\title{
Comparison of construction photograph and VR image in construction progress
}

\author{
Hyungeun Kim and Naruo Kano
}

\begin{abstract}
A photograph always makes an image of objects as they are without any alteration, so that a photograph is recognized as highly accurate information. Thus the photograph has been widely used as a record of site, routine work particularly for construction control and inspection. As a characteristic of photograph, the point of view for a photo image is identical to that of a VR image which can be created based on 3-dimensional CAD. In other words, matching of the photo image and the VR image provides a convenient way to examine the difference between the actual situation in a job site and the design drawing for the building.

This paper suggests the method for determination of the 3-dimensional viewpoint and the direction vector of a construction photograph to perform comparison of the construction photograph and the corresponding VR image. In addition, through a case study in which acquisition of construction photographs and comparison of construction photographs and VR images according to the elapsed time have been carried out, the validity of proposed methods has been well proved.
\end{abstract}

Keywords - Virtual reality, Progress control, Construction photograph, Image comparison

\section{INTRODUCTION}

$S_{\text {in }}^{\text {in }}$ ince the first employment of VR technology to construction industry in late 1980's, the use of VR(Virtual Reality) technology has expanded to construction control in practice by the virtue of rapid progress of personal computer. Simulation of construction progress in a VR space enables preliminary examination of construction methods or procedures by visual expression of construction progress that helps predict the problems during the actual construction. And a construction plan can be then provided based on the simulation. However, because it is difficult to perfectly expect construction situation

The research described in this paper was sponsored by a grant to WABOT-HOUSE project that was entrusted by Gihu Prefecture. This support is gratefully acknowledged.

Advanced Research Institute for Science and Eng., Waseda Univ., 3-4-1 Okubo Shinjuku, Tokyo, Japan(e-mail:kim@ aoni.waseda.jp)

Department of Architecture, Waseda Univ., 3-4-1 Okubo Shinjuku, Tokyo, Japan in the whole construction period prior to actual works in a job site, the details of construction plan are subject to change according to the situation of construction progress after the actual site works have already begun. Besides, the unexpected problems in the course of actual construction may impose modification of the construction plan. Therefore, exact information on the construction situation after the beginning of construction is indispensable for proper construction control. This study aims to compare the photo images of actual job site containing exact information on the construction situation with the planned VR images of construction simulated in VR space. Every object reflected in a VR space has its 3-dimensional coordinate, which makes it possible to see the object with a various view point as performing walk-through. But the 3-dimensional coordinate and direction of view point of construction photograph are required for the comparison with the VR image. This paper addresses acquisition of construction photographs in an actual job site and suggestion of the method for presuming 3-dimensional information on view points and directions of the construction photographs. Particularly it extends to providing a new method for construction control relying on comparison between the construction photograph taken at an actual job site and the virtual images corresponding to them. In addition, the validity of the proposed method for data acquisition and construction control has been estimated through case studies of actual job sites.

\section{CONSTRUCTION CONTROL THROUGH COMPARISON OF VR IMAGES AND CONSTRUCTION PHOTOGRAPHS}

\subsection{Virtual Construction Site(VCS)}

Using the 3-dimensional CAD enables grasp of time and space in construction industry. The 3-dimensional CAD is able to deal with information on building construction in a 3-dimensional space, so that an overall construction plan and control in a new concept can be provided by connecting time, object and activity to the information. Spatial shape, location and direction etc. for visible objects such as parts, members and workers are required to express building construction in a VR space. Again, it is necessary to figure out time-marching movement of those entities for expression of construction progress in the VR space.

As shown in Figure 1 the VCS system consists of a 3D CAD system, a VR system and a CG system. The 3D CAD system and the $\mathrm{CG}$ system mainly prepare information on building projects. 
The information is constructed depending on the purpose of 3D modeling and the accuracy in demand. The VR system presents visible recognition of situation in a construction site with real time walk-through.

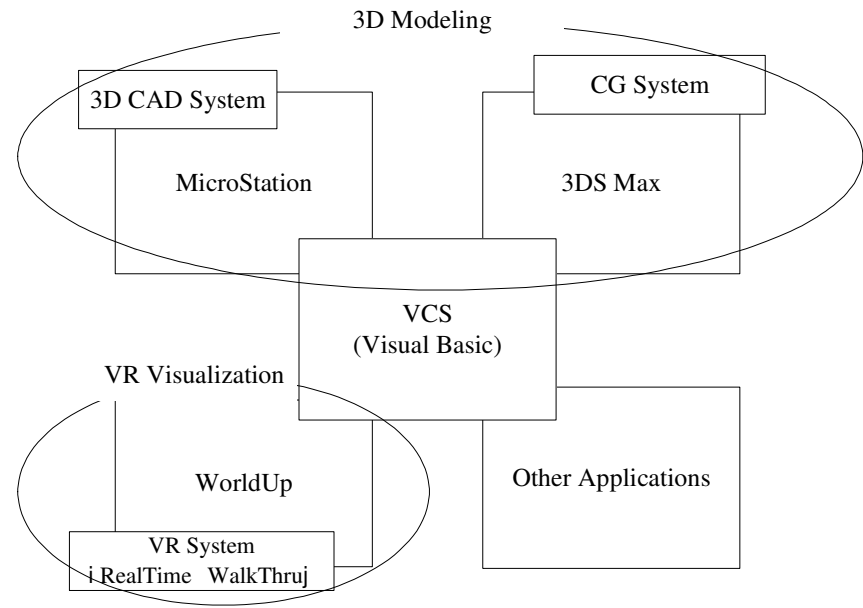

Fig.1. Basic concept for VCS system

\subsection{Comparison of construction photograph with VR image}

The construction photographs taken at various locations at an actual job site can be compared with the VR images created with the viewpoint and direction vector same as the corresponding construction photographs in virtual space. Comparing the two images with the identical 3D coordinate of viewpoint and direction vector enables examination of the difference between 3D CAD plans and actual situation in a job site with respect to the same objects such as the location or shape of building members. Furthermore, it facilitates visible recognition of the actual construction situation that differs from the simulated situation in the VR space. Figure 2 shows the visual comparison of a construction photograph to the corresponding VR image by superimposing the construction photograph on the VR image with adjusting transparency of the construction photograph.

\subsection{Aquisition of construction photographs}

Photo taking at an actual job site provides a very useful tool for the record of construction progress and the inspection of built-up members in the aspect that it can provide unsuspicious data for a certain situation. And the construction photograph itself is dealt with as important information with respect to product control such as grasp of construction progress and quality inspection. The construction photographs for various activities in a job site are taken every day with an accurate coordinates for them which can be suggested by total station. The acquisition of exact coordinate is strongly necessary to correctly match the photos with the images from VR space. As for camera used in a job site, wide range of camera from normal digital camera to web camera has been used according to the method of data acquisition. Some cameras provide a convenient function that successive photos in a long term can be automatically taken with identical location and direction.

\subsection{D Information on viewpoint of construction photograph}

In order to compare a construction photographs to a VR image, the viewpoint and direction vector of two images should be coincide. Each construction photograph requires the viewpoint and direction vector. According to the purpose and accuracy of construction photograph, the following three methods for determining the $3 \mathrm{D}$ viewpoint of a construction photograph can be suggested.

1) Determination of $3 \mathrm{D}$ viewpoint by survey of actual object

2) Determination of $3 \mathrm{D}$ viewpoint by analysis of photo image

3) Determination of 3D viewpoint by survey of location of fixed-point camera

This paper performs the comparison of construction photograph holding 3D viewpoint determined by survey of fixed-point camera and the VR image holding the identical viewpoint to the photo.

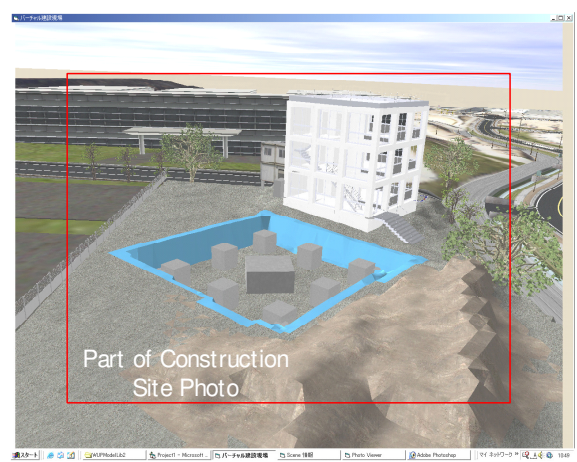

a) Transparency of construction photograph; $100 \%$

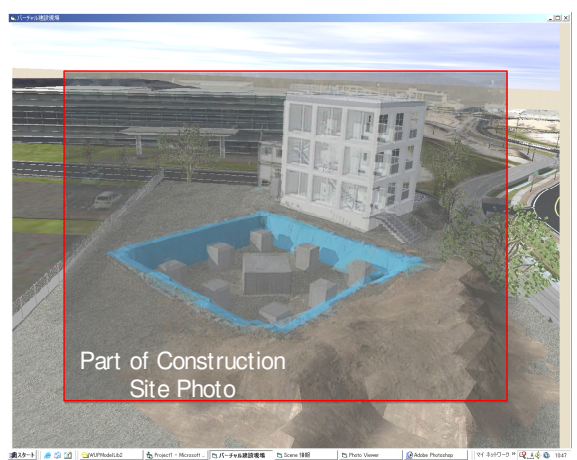

b) Transparency of construction photograph; $50 \%$

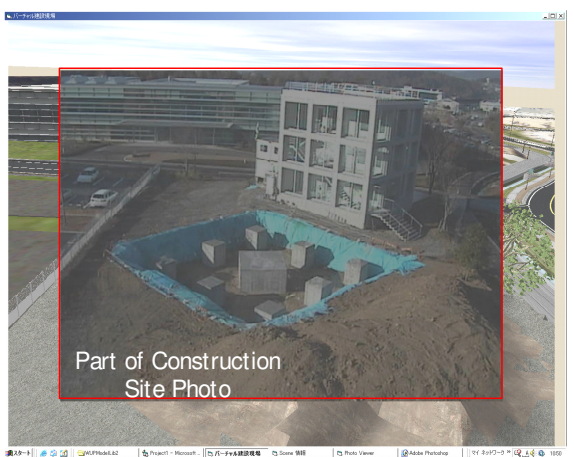

c) Transparency of construction photograph; $0 \%$

Fig.2. Control of transparency of construction photograph in comparison with VR image 


\section{DETERMINATION OF 3D VIEWPOINT BY SURVEY OF LOCATION OF FIXED-POINT CAMERA}

\subsection{D viewpoint of fixed-point camera}

A construction photograph is taken at various location and time in an actual job site. To collect proper construction photographs efficiently, the use of appropriate camera is required according to the purpose and location for photo taking. And the coordinate of $3 \mathrm{D}$ viewpoint of fixed-point camera can be obtained by surveying the location of the camera using a surveying apparatus as shown in Figure 3. The direction vector of the photo is obtained using VCS on the basis of target point which is set to the center of construction photograph. This study performed collection of construction photographs using fixed-point camera according to the purpose of construction photograph.
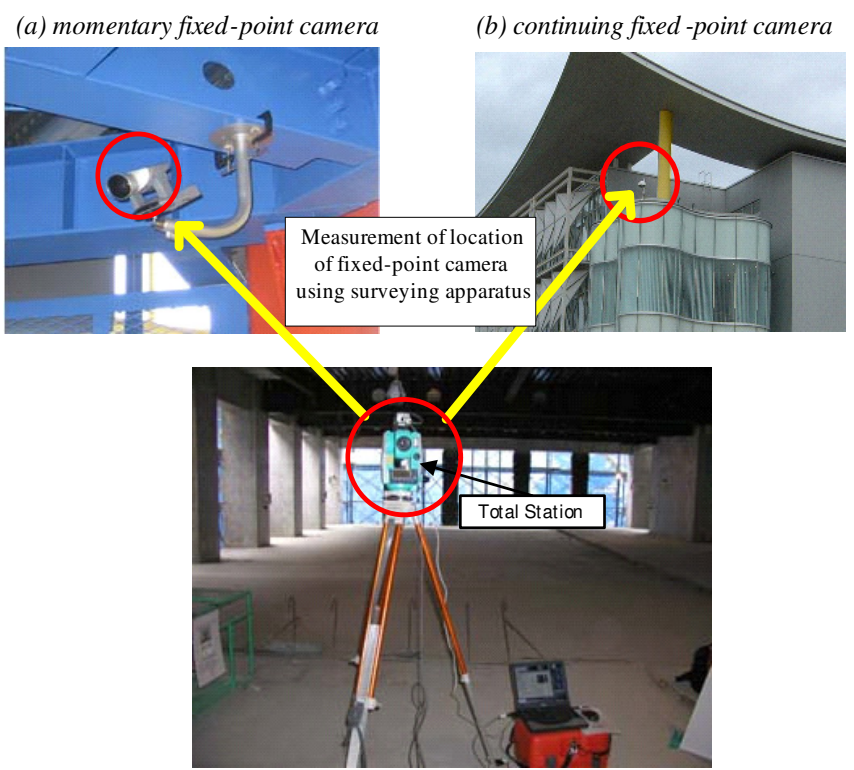

Fig.3 Determination of 3D viewpoint by survey of location of fixed-point camera

\subsection{Continuing fixed-point camera}

As shown in Figure3 (b), the continuing fixed-point camera is readily located at around a construction site with few its relocation until the end of construction. The continuing fixed-point camera has advantage of taking photos of same direction automatically at the same location everyday. Therefore, it makes it easier to examine the time-marching situation of current construction visually using the construction photographs taken with the same viewpoint and direction vector.

\subsection{Momentary fixed-point camera}

From time to time, photo taking at around a job site might be obstructed owing to safety net which is built up along the boundary of a job site before the beginning of construction responding to some particular requirements for safety. Again, it is inevitable to locate a camera inside a job site to acquire construction photographs containing details inside the job site. However, the locations for camera should be selected to avoid obstruction of workers inside the job site. Photo-taking is meaningless at the locations in which all the construction works has been completed, so that the camera will be frequently moved to more appropriate places. For that reason, a portable digital camera or web camera is well used for momentary fixed-point camera as shown in Figure 3 (a).

\subsection{Correction of horizontal angle of camera in construction photographs}

In spite of the importance of fixing a camera to a tripod or rack precisely for accurate comparison of a construction photograph and a VR image, a small amount of rotational deviation of the camera is unavoidable. For the sake of correction of such rotational deviation, the construction photograph is adjusted to coincide with the corresponding VR image by rotating itself to the deviation angle, $\theta$ with the center at the targeting point from VCS. Figure 4 illustrates the inclined wall of building and temporary fence in the white circles before correction in the

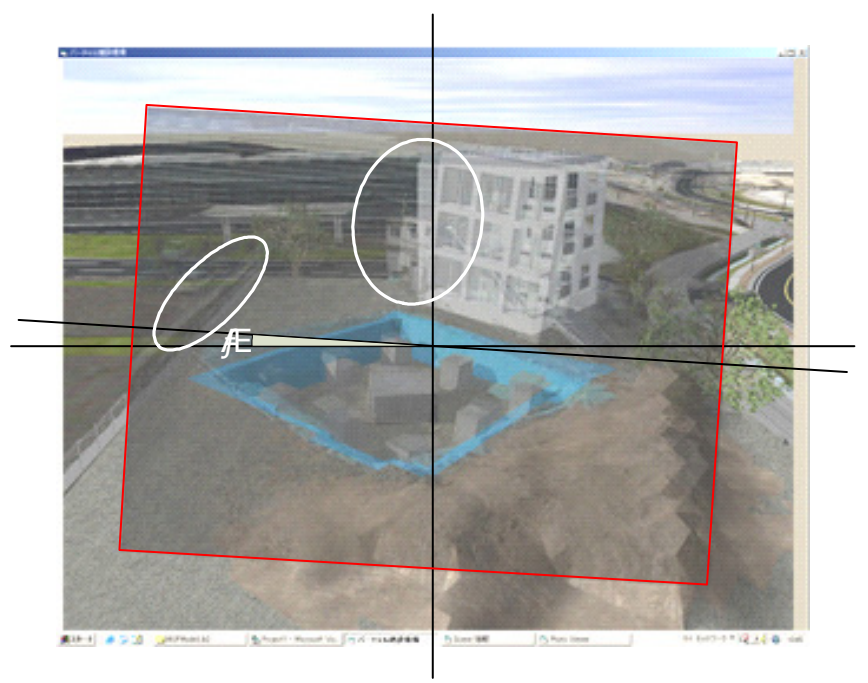

a) Before correcting the deviation angle

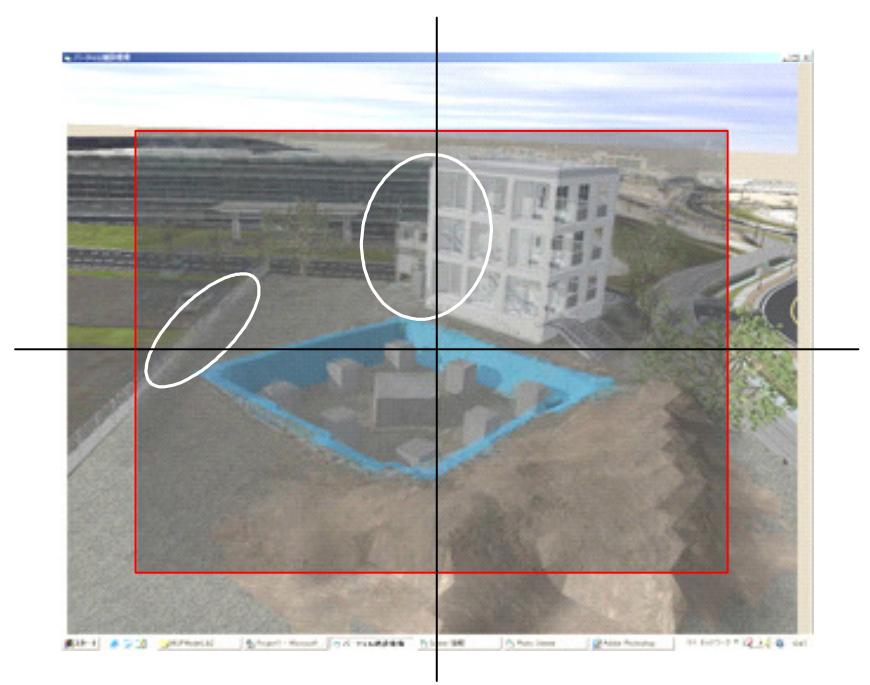

b) After correcting the deviation angle

Fig.4. Correction of deviation angle for superimposing of construction photograph on VR image 
construction photograph, which is seen to completely coincide with the VR image after correction.

\section{CASE STUdy}

\subsection{Adopted Model}

As for 3D modeling, the model for drawing information requiring high accuracy was constructed using the "Micro Station" which is one of the 3D CADs, whereas the model for building materials and machineries requiring somewhat less accuracy was using the "3DS Max." And the modeling of site was created with enhanced accuracy in such a way that the modeling reproduced the levels of buildings and sites even on the preliminary layout. Besides, the modeling for the surrounding buildings excluded from the layout was implemented based on the surveyed data. The construction photographs were compared to the simulated images of construction progress in VR space that had been generated based on the 3D modeling. The outline of object construction site is summarized in Table1. Figure 5 displays a portion of time schedule.

Table1. Outline of construction

\begin{tabular}{|c|c|}
\hline Project Name & Gihu Prefecture Robot Plaza Cbuilding \\
\hline Usage & Robot research facilities \\
\hline Lot Area & 再田, $245.51 \mathrm{~m}^{2}$ \\
\hline Architectural Area & 田田50.063 $\mathrm{m}^{2}$ \\
\hline Total Floor Area & 田 $132.0 \mathrm{~m}^{2}$ \\
\hline Number of Stories & Fifth Floor \\
\hline Starting Day & 酉的004/11 \\
\hline Ending Day & 再目005/4 \\
\hline \begin{tabular}{|c|c|c|c|c|c|c|c|c|}
2 & 3 & 4 & 5 & 6 & 7 & 8 & 9 & 1 \\
ed Thu & Fri & Sat & Sun & Mon & Tue & Wed & Thu & F
\end{tabular} & 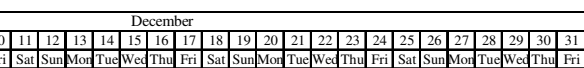 \\
\hline $\begin{array}{l:l:l} & \vdots & \vdots \\
\end{array}$ & 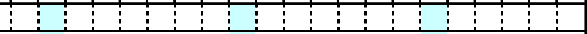 \\
\hline 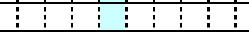 & 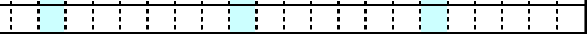 \\
\hline imud salb : $\quad$ feefin & g concrete $\quad$ ianchor: $:$ ibase concrete \\
\hline 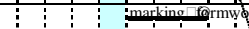 & $\begin{array}{c:c}1 \text { marking } \\
\end{array}$ \\
\hline $\begin{array}{c:c:c}\vdots & \vdots & \vdots \\
\vdots & \vdots\end{array}$ & iback filling iformwork \\
\hline$:$ & : \\
\hline 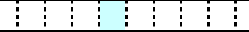 & 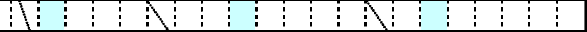 \\
\hline$\vdots$ & $\vdots \vdots$ \\
\hline$:$ & $1: \quad:$ \\
\hline : & $\begin{array}{l:l:l} & 1 & 1 \\
1 & 1 & 1\end{array}$ \\
\hline a portion of & fonting concrete \\
\hline \begin{tabular}{|lll} 
& delayed work \\
\end{tabular} & : $\quad$ backfilling \\
\hline $5: \quad: 5:$ & 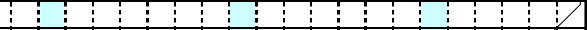 \\
\hline 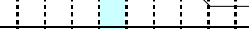 & 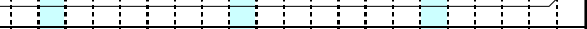 \\
\hline $\begin{array}{ll:l:}1 & 1 \\
\end{array}$ & 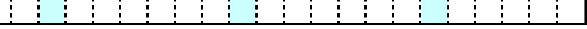 \\
\hline
\end{tabular}

Fig.5. A portion of time schedule for a construction project

\subsection{Acquisition of construction photographs}

As the actual construction began, the construction photographs had been acquired through photographing daily situation of construction progress automatically with an interval of one hour during the working time, from $7 \mathrm{am}$ to $5 \mathrm{pm}$, by the use of a continuing fixed-point camera. Furthermore, using a web camera for the continuing fixed-point camera facilitates monitoring the actual daily construction progress on web. As scaffolds were built up, a momentary fixed-point camera as well as the continuing fixed-point camera began to provide construction photographs. The coordinate of viewpoint of the continuing fixed-point camera was surveyed as $(55.887,13.652$, 14.030) in $\mathrm{x}_{\mathrm{v}}, \mathrm{y}_{\mathrm{v}}, \mathrm{z}_{\mathrm{v}}$, and the direction vector of the camera was determined as $(-0.82766,-0.44959,-0.33589)$ in $x_{d}, y_{d}, z_{d}$ from VCS. Besides, the coordinate of viewpoint of momentary fixed-point camera was surveyed at every time the location of camera changed.

\subsection{Construction control through comparison of construction photograph to VR image}

First, the construction photographs acquired from both the continuing and momentary cameras were compared to the images planned in VR space regarding the construction situations until the middle of March including the in-situ situation before the beginning of construction. Figure 6 illustrates several sets of the two images comparing the situations of construction progress corresponding to date. Most sets of the images show good comparison between the construction photographs and the VR images according to the schedule chart.

As illustrated in Figure 6, the two images, dated 28-Nov-2004, is showing the temporary fences and the temporary building for office as preparations for the main construction, and the set of images on 5-Dec-2004 shows the excavation of foundation ground executed up to $80 \%$ of its whole process. The images planned in VR space indicate accomplishment of the foundation at 9-Dec-2004. However, the construction photograph dated 13-Dec-2004 displays the same situation. Even though the VR image dated 14-Dec-2004 illustrates the situation for accomplishment of refills, the situation is seen to have appeared at the actual job site on 20-Dec-2004. Although the marking with black ink was planned to be completed at 27-Dec-2004 on the construction schedule for December, 2004 as shown in Figure 5, the construction photograph taken at 28-Dec-2004 shows that the situation exhibiting execution of lean concrete corresponded to the image planned at 22-Dec-2004 in VR space, which indicates that the actual construction progress was delayed by 6 days compared to the schedule. Nevertheless, because the curing of pit base concrete was scheduled until 5-Feb-2005, the 6-day delay hardly affected the entire period of construction. On 26-Jan-2005, scaffolds were set up around the site, then it appeared from 5-Feb-2005 that steel erection could commence according to the construction schedule.

Besides, since the scaffolds were obstructing the view of continuing camera located at around the site, the construction photographs acquired using the momentary fixed-point camera were compared to the VR images after 3-Feb-2005. The construction photographs taken with the momentary fixed-point camera hold diverse viewpoints and direction vectors depending upon the date and the location for photographing. The photo dated 3-Feb-2005 shows the situation that concrete was completely placed at pit base. Steel erection started from 5 -Feb-2005, the photo taken at 8-Feb-2005 displays the situation in which small be a m w a s 


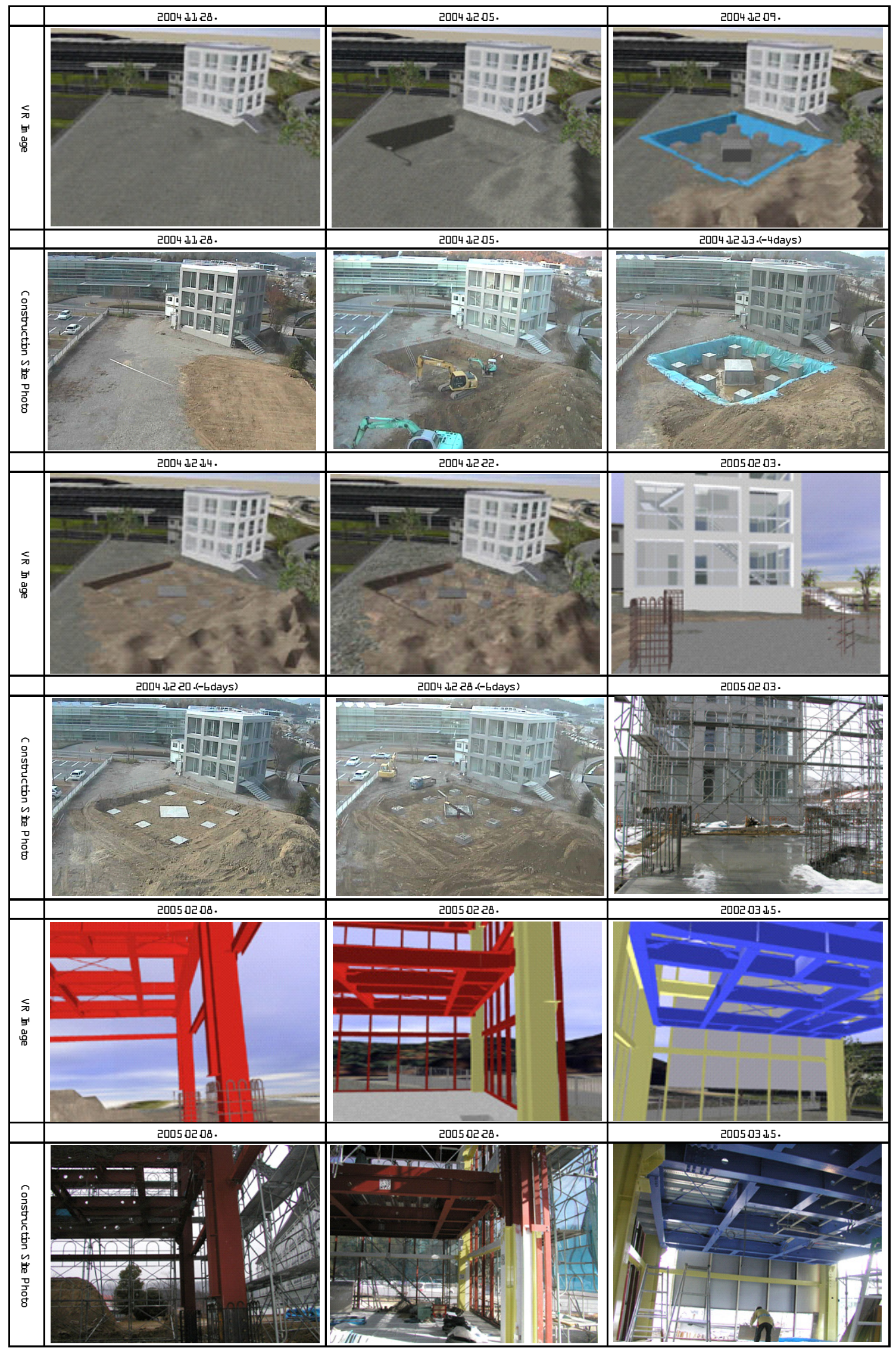

Fig.6. Comparison of VR images and construction photographs acquired using fixed-point camera 
installed on the floor. The situation in the photo taken at 8-Feb-2005 includes placement of slab concrete and beams for ground floor and partial painting of members in steel frame. Again, facility work began at 14-Mar-2005, and it appeared at site that panel work regarding had been carried out continuously for three days. However, installation of curtain wall on the schedule was not seen at the site. The construction photograph dated 15-Mar-2005 shows that installation of panel was performed up to $60 \%$ of its whole process, the forms for the concrete beams in the ground floor was in the process of disjointing. Considering the construction progress on the basis of the work schedule and nonexistence of curtain wall, which was the critical path, at the construction photographs dated 15-Mar-2005, a 5-day delay of entire period of construction would be expected.

\subsection{Summary}

The VR image with the direction and location same as those of the construction photograph taken with fixed-point camera naturally reflects nearly the identical region to that of the construction photograph. The comparison of these two images revealed that the location and shape of building members in an actual job site could be compared to the design drawings created by $3 \mathrm{D}$ CAD. It was also confirmed that the VR images simulating the construction progress in VR space could be visually compared to the situation of actual construction site expressed by the construction photographs. Consequently it was found that the comparison of the situations in VR space and at an actual job site enables acquisition of useful information with feedback on the planning of construction process.

\section{CONCLUSION}

With a point of view to importance of visual decision making at an actual construction site, this paper presents the new construction control method employing the construction photographs that were taken with a fixed-point camera as exact information on actual situations in a job site and the VR images which were created using VR technology. To determine the $3 \mathrm{D}$ viewpoint of the construction photograph, the determining methods for the coordinate of viewpoint and the direction vector of a construction photograph acquired from an actual job site have been suggested. Through a case study of building construction, it is successfully proved in this research that comparison between construction photographs and VR images is valid for the visual assessment of construction progress in a job site. Furthermore, for the sake of rather exact comparison of VR images and construction photographs, correction of the deviation angle of camera in horizontal plane and the $3 \mathrm{D}$ viewpoint of a construction photograph has been presented so that rather accurate matching of construction photographs on VR images can be well achieved.

\section{REFERENCES}

[1] Naruo Kano, etc., "Virtual Construction Site Development of a Prototype System and the Issues", Building Construction and Management of Projects, AIJ, Vol.18, pp67-72, 2002.07
[2] Naruo Kano, etc., "Virtual Construction Site; Visualized Simulation for Construction Site", Building Construction and Management of Projects, AIJ, Vol.19, pp95-102, 2003.07

[3] Hyungeun Kim, etc., "A Study on the Application of VR Technique to Building Construction; Comparisons of Site photo and VR Image in Construction Progress, Computer Technology of Information, AIJ, Vol.27, pp31-36, 2004.12

[4] Hyungeun Kim, etc.," A Study on the Application of VR Technique to Building Construction", ISARC2004(Korea), pp431-436, 2004.9 